\section{MASCHERE FEMMINILI VERGHIANE TRA NARRATIVA E} CINEMA

VERGA'S FEMININE MASKS BETWEEN FICTION AND CINEMA

Dora Marchese

Universidad de Catania

RIASSUNTO:

ABSTRACT:

Nel presente articolo si analizza la donna In this article Verga's women are analized. verghiana, considerata una maschera talvolta Sometimes they can be considered sentimental sentimentale e coraggiosa, altre volte femme and brave, other times femme fatal. Aglea is fatal. Emblema delle maschere verghiane è an emblem of Verga's masks, she symbolizes a considerata Aglea, figura di femme fatal che femme fatal that will be sarcastically reversed. poi sarcasticamente ribaltata.

PAROLE CHIAVE:

KEY wORD:

Maschera, donna, Verga.
L'etimo del termine "maschera" è incerto. Tra le ipotesi più accreditate quella della provenienza dal latino medioevale "màsca", "strega", tuttora utilizzato in tal senso nel piemontese. Altre tracce si riscontrano nell'antico alto-tedesco (leggi longobarde) e nel provenzale "masc", stregone. Dal significato originario si giunse successivamente a quello di "fantasma", "larva", "aspetto camuffato volto ad incutere paura". Secondo gli studiosi, l'evoluzione linguistica portò probabilmente all'aggiunta di una " $r$ " facendo assumere al termine la forma dapprima di "mascra" e successivamente di "mascara". In senso figurato, l'espressione è sinonimo di finzione, apparenza, immagine, impostura simulazione. La rigida fissità e l'ipocrisia (ricordiamo del resto che "hupokrit?s" in greco significa "attore") sono dunque qualificanti del concetto e dell'immagine stessa di "maschera".

Questa considerazione preliminare ci giova per suggerire come alcune delle protagoniste femminili della produzione letteraria verghiana della giovinezza e della maturità possano a buon diritto essere considerate appunto maschere, figure di donne tutte di un pezzo, altere, monocordi, spesso prive di una variegata e complessa caratterizzazione psicologica. Già ne I Carbonari della montagna Verga traccia il personaggio di Giustina quale fanciulla appassionata, sentimentale e pura, fedele all'uomo che ama, animata da amor patrio, coraggiosa e intrepida, in contrapposizione a Carolina, sciagurata e corrotta donna-demonio. Giustina assomma in sé tutte le caratteristiche peculiari delle eroine della coeva letteratura e del melodramma d'epoca risorgimentale, anticipando un'iconografia che ritornerà nel successivo Sulle lagune in cui l'anticonformismo di Giulia, il suo atteggiamento audace e istintivo, ricordano molto da vicino le caratteristiche della volitiva protagonista del primo romanzo dello scrittore. Ma è da Tigre reale in poi che Verga proporrà la maschera della femme fatale in netta contrapposizione a quella di angelo del focolare.

Ovviamente la prima tipologia è presente in modo preponderante nelle opere della gioventù le quali, essendo di ambientazione aristocratica e alto-borghese, pongono sotto i riflettori donne affascinanti, conturbanti, vanitose, spesso crudeli. Una tipologia in cui, val la pena ricordarlo, influì non poco, oltre alle opere di Sue, anche il modello della Milady di Dumas, autore conosciuto e stimato da Verga che gli inviò una copiaomaggio de I Carbonari della montagna.

Protagonista di Una peccatrice, Narcisa al fascino perverso, irresistibile e funesto di Carolina, coniuga, agli occhi del siciliano Pietro, l'elemento dell'esotismo e della estraneità geografica e emozionale. Non bella secondo i canoni convenzionali ma ammantata di un mistero "razionalmente inspiegabile, indicibile", è un'aristocratica, continentale, ambigua, la maschera stessa dell'inaccessibilità, un idolo, un'icona. Donna di lusso, artificiosa e impassibile, è descritta da Verga con toni eccessivi e melodrammatici, facendo ricorso a puntuali descrizioni inerenti il trucco, la toilette, 
l'abbigliamento. "Io amo nella donna" - confessa Pietro - "i velluti, i veli, i diamanti, il profumo, la mezza luce, il lusso". A metà tra mito e natura, Narcisa è "silfide", "maga", "sirena", "fata", è "fiore" e "farfalla". Anche in Tigre reale, Giorgio La Ferlita si esprime in modo analogo: "Caro Bassano, la donna è un oggetto di lusso, quando potrò permettermi sei cavalli in scuderia, invece di due, allora mi regalerò un'amante". Quando la sua maschera si umanizzerà e la contessa di Prato si concederà all'amore di Pietro Brusio l'incantesimo sarà spezzato per sempre. La conquistata umanità ne distrugge il simulacro, l'essenza stessa, la pulsione profonda capace di affascinare ed imprigionare l'uomo in preda ad un perenne desiderio inappagato. Pietro ne è perfettamente consapevole:

Ti svelo un gran mistero del mio cuore, che Narcisa non dovrebbe mai conoscere. In mezzo a questi deliranti piaceri, in mezzo a questa felicità $[\ldots]$ penso che $[\ldots]$ a forza d'immedesimarmi nella vita di lei, a forza di assorbirne tutte le emanazioni [...] io non giunga a rompere quel velo aereo, direi, di cui Narcisa si circonda, e che comanda quasi la semioscurità, l'isolamento, per farla meglio ammirare.

Obbedendo ai canoni più abusati, secondo norma le eroine 'irregolari' espiano duramente la loro anticonvenzionalità cessando di vivere o scomparendo del tutto dalla scena. Narcisa muore e Pietro trova conforto presso la famiglia, in Sicilia. Ma rispetto alla dama delle camelie di Dumas o alla Violetta verdiana, la protagonista del racconto di Verga è caratterizzata proprio dalla fissità, dall'artificiosità, dall'essere presentata come miraggio, illusione: "io l'amo come un bel personaggio da dramma e da romanzo", dirà ancora Pietro. Non a caso, l'ambiente prediletto in cui si muovono Narcisa, Nata ed Eva è proprio quello teatrale, quello delle feste e degli spettacoli, in una speculare e reciproca rifrazione fra l'alta società e l'effimero mondo del teatro.

Accanto al 'travestimento' realizzato per mezzo di trucchi, gioielli e abiti fastosi, sovente è raffigurato il travisamento del carnevale, in cui le maschere femminili verghiane si caricano di ulteriori pregnanti significati. Il ballo in maschera durante il giovedì grasso rappresenta un momento importante nello svolgimento della storia di Pietro e Narcisa; Eva prende abbrivio con una scena carnascialesca, il veglione al Della Pergola che introduce il lettore in medias res per poi ricondurlo, tramite flashback, alle origini della passione di Enrico Lanti per la seducente donna. Preludio dell'imminente quaresima, il carnevale diviene l'estremo trionfo dell'eros prima del definitivo affermarsi del thanatos, un momento di ambiguità in cui la sfrenata e forzata allegria si vena di sfumature malinconiche e mortifere. Anche nella novella Di là del mare, compendio ed explicit delle Novelle rusticane, il carnevale assume un aspetto luttuoso e sinistro. I due amanti, lui siciliano, lei continentale, dopo una lunga separazione infine si rivedevano nella vertigine del carnevale. Egli era andato alla festa per veder lei, coll'anima stanca e il cuore serrato d'angoscia. Ella era lì difatti, splendente, circondata e lusingata in cento modi. Pure aveva il viso stanco anch'essa, e il sorriso triste e distratto. I loro occhi s'incontrarono e scintillarono. Nulla più.

L'esotismo delle immagini di donne feline largamente rintracciabili nei romanzi fiorentini (da Una peccatrice in cui Narcisa morente appare come una tigre urlante per lo spasimo e il dolore dell'abbandono, a Tigre reale - originariamente Felis mulier - in cui ampi e ricorrenti sono i riferimenti alla donna-tigre o leonessa), in seguito alla maturazione artistica scompare, mutandosi in più consueti paragoni e in immagini di gran lunga più efficaci e incisive. Alla fascinosa e preziosa donna tigre, leone, giaguaro, ostentante un atteggiamento frigido ed artificioso, subentra la terragna e sensuale donna "lupa", tutta natura e istinto, che solo in apparenza pare conservare le peculiarità della maschera impenetrabile, inamovibile, determinata e impavida delle eroine dei lavori giovanili. La vicenda amorosa di questa creatura primordiale che "segue il ciclo vitale della natura" racchiude in sé "l'agonia dell'esperienza dell'esistere". Così la Lupa, pur scorgendo la scure di Nanni Lasca "che luccicava al sole", "non si arretrò di un sol passo, non chinò gli occhi, seguitò ad andargli incontro, [...] mangiandoselo con gli occhi neri". Così Peppa, l'amante di Gramigna, alle domande preoccupate della madre "non rispondeva neppure, colla testa bassa, la faccia dura, senza pietà per la mamma che faceva come una pazza, coi capelli grigi al vento, e pareva una strega". La ragazza rincorre e rintraccia Gramigna, che se la vede venire incontro "risoluta, in mezzo alle macchie fitte, nel fosco chiarore dell'alba", senza mai esitare nel seguirlo nétra le campagne in mezzo a "i fichidindia di Palagonia", né in città "dove le avevano detto ch'era in carcere Gramigna", dopo aver lasciato "il figliuolo ai trovatelli, senza voltarsi indietro neppure". Dalla pagina allo schermo, le maschere delle protagoniste verghiane di Una peccatrice, Tigre reale, La Lupa e L'amante di Gramigna si arricchiscono di nuove connotazioni, si trasformano piegate dall'ideologia politico-sociale, dai gusti e dalle necessità del mercato, talvolta semplicemente dall'ignoranza o dalla noncuranza nei confronti della poetica verghiana.

Girato da Piero Fosco (Giovanni Pastrone), di Tigre reale del 1916, con Pina Menichelli (contessa Nata), Alberto Nepoti (ambasciatore Giorgio La Ferlita), Valentina Frascaroli (Erminia), Gabriel Moreau (conte de Rancy), la critica dell'epoca mette subito in evidenza che, diversamente dal romanzo di Verga in cui la contessa appare "un essere strano, bisbetico, malato, viziato e insensibile, dolce e terribile, che carezza e schiaffeggia, che bacia e morde", viceversa nel film Natka è "una donna normale, profondamente innamorata del suo amante, con qualche lieve stranezza". Non solo, ma il cronista di "Cine-Fono" continua sottolineando la gravità della totale "evirazione" subita dal personaggio della moglie di La Ferlita, Erminia, che se ha reso possibile far risaltare pienamente la primadonna Menichelli e giungere facilmente all'happy end, in sostanza tradisce non soltanto la lettera ma anche lo spirito del romanzo. Nell'opera di Verga, 
infatti, gli affetti familiari, quelli per la moglie e per il figlio, la coscienza della sacralitàde matrimonio, sottraggono Giorgio dalla perdizione totale ed irreversibile, lo salvanodal baratro in cui rischia di sprofondare. Nonostante il suo bambino corra pericolodi vita Giorgio, pazzo di passione per la contessa, lo abbandona per poter trascorre insieme a le una "orribile notte d'amore che sembrava durare eterna". Sola, spaventata, disperata, Erminia trova conforto nel cugino Carlo cui è legata da antichi, inespressi sentimenti. Tra il marito traditore e menefreghista, assente nel frangente più dolorosodella sua vita, e il cugino disposto a tutto compiere per lei, questa donna, simbolo di purezza, onestà e virtù, sceglie di riaccogliere il legittimo sposo e di allontanare per sempre Carlo, incarnando in modo decisivo il ruolo della sposa fedele, incorruttibile, pietosa e comprensiva. Non solo Ma anziché attendere una dichiarazione di pentimento da parte del marito, è lei a confessargli: "Ho amato Carlo!". Giorgio, a questo punto, "non le disse una sola parola, si chinò sul letto, la abbracciò stretta, colla fronte su quella di lei, e confusero insieme le loro lacrime". In seguito, alla stazione dei treni, turbato dalla visione del convoglio che conduce all'ultima destinazione la bara di Nata,Giorgio, che sembra nuovamente in preda al tremendo maleficio, è ancora consolato da Erminia: "Povero Giorgio [...] noi ti ameremo tanto! Tanto...". Ridotta Erminia a semplice comparsa, nel film di Pastrone non c'è traccia di tale complessa e pregnante vicenda.

Erminia è promessa sposa a Giorgio che, tuttavia, la abbandona prontamente poco prima delle nozze, totalmente avvinto dall'incantesimo della contessa russa: "civetta orgogliosa, egoista, marmo di Carrara dentro e fuori [...] sorriso glaciale". Il critico Pio Fasanelli osserva a tal proposito: "chi ha letto il romanzo ha dovuto notare lo stato d'animo di Erminia, nel vedersi trascurata da suo marito e corteggiata dal cugino, le atroci sofferenze di Giorgio roso dalla gelosia per l'assidua presenza del tenente Carlo presso la moglie, e dal rimorso di tradire l'Erminia pur non sentendo la forza di staccarsi da Nata. Sono tutti questi contrasti dell'anima umana che andrebbero rilevati in certi films, tessuti di profonda psicologia. Ma nella Tigre reale si aveva in animo di poggiare tutto il lavoro sulla "primadonna'".

Francamente poco credibile, poi, nella pellicola di Pastrone, il lieto fine in cui i due amanti scampano al fuoco appiccato dal marito di Nata geloso che li chiude a chiave in una stanza d'albergo. Nonostante sia indebolita dall'incipiente tubercolosi, trovata prontamente una barca con cui fuggire "nello splendore del tramonto di fuoco..., Nata fremente di amore per Giorgio adorato si sente miracolosamente tornare alla gioventù e alla vita".

Se nel romanzo, dunque, vince la donna-angelo, Erminia appunto, dolce, paziente, comprensivo porto sicuro dalle procelle funeste della passione, nel film vince la femme fatale, la donna luciferina Nata. Lo stravolgimento del finale di Tigre reale, il cui soggetto era stato ceduto dallo scrittore siciliano a Dina Castellazzi di Sordevolo insieme a quell di Una peccatrice ed Eva, viene del resto così commentato da Verga: "Io non mi oppongo. [...] Tanto nel cinematografo se ne vedono tante, e tante me ne han fatto vedere e ne vedo". Quando due anni più tardi (1918) appare Una peccatrice di Giulio Antamoro, con Leda Gys (Narcisa Valderi, contessa di Prato), Goffredo D’ Andrea (Pietro Brusio), la critica è ugualmente dura:

Riduzione e sceneggiatura del soggetto dal romanzo del Verga: ignobile. [...] È una volgarità sola, dal principio alla fine. Una signora delle camelie riveduta e scorretta. [...] Dell'interpretazione, meglio non parlarne! Vecchi giuochi, maniera, falsità, nessuna sincerità. Le toilettes della Gys sempre indossate fuori era: troppi décolletés, troppi scarpini!

Riguardo Eva (1919), regia di Ivo Illuminati, con Alba Primavera (Eva) e Attilio De Virgilis (Enrico Lanti), se l'accoglienza di critica e pubblico è tiepida (“Un lavoruccio simpatico, in cui se non vi è nulla da ammirare, non v'è neppure nulla da disprezzare"), le parole di Verga a Dina in una lettera del 7 giugno 1918 sono colme di rassegnazione: “Ahimè, amica mia! Questo Cinematografo è un castigo di Dio [...]. Se sapeste quanti sopraccapi e preoccupazioni e dispiacieri, in mezzo a tanti altri che ci ho di tanti altri generi!". Un discorso a parte meritano le trasposizioni da La Lupa e L'amante di Gramigna.

Ambientato a Matera, La Lupa di Alberto Lattuada (1953) ha protagonista la conturbante Kerima, sin dal suo primo apparire presentata come una donna libera e indipendente, fortemente volitiva. Anche se la critica non accolse con entusiasmo la prova di Lattuada, riconoscendogli una certa perizia di mestiere ma rimproverandogli una vicinanza allo stile fiorito di d'Annunzio più che a quello scabro di Verga, pure una felice intuizione si trova nell'incipit allorché la Lupa si reca da don Pietro per convincerlo ad assegnare alla figlia Maricchia (May Britt) il ruolo di Sant'Agata nella processione per la patrona. Il contrasto tra l'ingenuità e la purezza di Maricchia e la prepotente sensualità della Lupa che si serve del suo fascino demoniaco per ottenere ciò che vuole ripropone la dicotomia donna-angelo / donna-diavolo presente non solo nella novella ma anche nel testo teatrale di Verga.

Se l'explicit della novella, di straordinaria potenza evocatrice, è: "non si arretrò di un sol passo, non chinò gli occhi, seguitò ad andargli incontro, con le mani piene di manipoli di papaveri rossi, e mangiandoselo con gli occhi neri"; nelle "Scene drammatiche in due atti" le didascalie descrivono la gnà Pina "colla bocca amara [...], sarcastica [...], dura e ostinata [...], guardandolo fisso in tono di amarezza disperata [...], voltandosi verso di lui, col petto nudo,come a sfidarlo", fino al sibilare delle ultime parole: "Finiscila! via! colle tue mani!". Il vieto topos del fuoco purificatore conclude, invece, la fosca vicenda nel film di Lattuada, allorché la Lupa si barrica nell'edificio della manifattura tabacchi 
e, di fronte a Nanni e Maricchia, a don Pietro, ad una folla intera che le inveisce contro, appicca l'incendio non tralasciando, tuttavia, anche in questo caso, di pronunziare ultime sprezzanti parole di sfida, scevre da ogni pentimento: "Venitemi a prendere se potete (mentre viene inghiottita dal fuoco) [...] venitemi a prendere...".

La versione del 1996 di Gabriele Lavia, con Monica Guerritore (gnà Pina), Raul Bova (Nanni Lasca), Alessia Fugardi (Maricchia), Giancarlo Giannini (padre Angiolino) e Michele Placido (Malerba), pur essendo un mediocre prodotto commerciale, mantiene anch'essa la nodale contrapposizione donna demone / angelo del focolare, imprescindibile per lo svolgersi della fabula. Per il resto, di questo esperimento poco riuscito rimangono "i paesaggi, la terra e le rocce bruciate dal sole accecante della Sicilia. A suggerire il tormento umano, reso ancor più melodrammatico dal rutilante commento musicale di Morricone".

Più pregevole la trasposizione cinematografica de L'amante di Gramigna di Carlo Lizzani (1969), con Gian Maria Volontè (Gramigna) e Stefania Sandrelli (Gemma), il cui intreccio, all'insegna della polemica meridionalistica e dei fermenti del '68, ha un tono epico, contrassegnato da uno stile essenziale e asciutto che riscosse notevole successo di critica e di pubblico. Emblematiche le parole con cui Gramigna cerca di scrollare i contadini paralizzati e attoniti: "Invece di sospirare, soffiate, ché se soffiamo tutti il vento si porta tutti i baroni del mondo!".

La protagonista è assai diversa dalla Peppa verghiana: “in Peppa non c'è rifiuto di un ruolo storico asservente, non c'è la dignità femminile e proto femminista che pure agita Gemma, non c'è lotta individuale per la conquista dei propri diritti come giusto riconoscimento della propria individualità. [...] Gemma rivendica, Peppa si lascia andare solamente all'istinto, all'animalità, alla sessualità", come la Lupa. E, come la Lupa, va incontro a Gramigna "risoluta", con lo sguardo "fisso", rispondendo alla proposta del bandito di seguirla: "Sì [...] col capo avidamente, sì". Contrapponendosi alla cieca e, almeno apparentemente inspiegabile, infatuazione di Peppa, Gemma s'infiamma progressivamente per Gramigna, sin dall'iniziale scena dell'iniqua estorsione della casa e del terreno suoi e del padre da parte del barone Eugenio Nardò. Un sentimento che nasce dall'ammirazione per il coraggio, il senso di giustizia, la volontà di riscatto del bandito. Un'intesa destinata a lievitare e a maturare fino a trasformarsi in amore reciproco e profondo, ricco di tenerezza e complicità. Positivo, fra gli altri, il commento di "Momento Sera": "Lizzani si è accostato al realismo di Verga con grande abilità, Volonté con rabbia, Stefania Sandrelli con la sua femminilità animalesca. Il risultato di questa felicissima combinazione di fattori è un film duro e amaro, preciso nei contorni come un dagherrotipo trovato in soffitta e lirico, poetico, straziante".

Il "mito del muliebrismo", per usare un'espressione di Russo, è destinato a fallire in Verga, mostrando le debolezze delle due facce dell'eros: l'angelica e la diabolica.
Nedda, Mena, Diodata, Bianca, Isabella, sono donne remissive e rassegnate, del tutto subalterne. Carolina, Narcisa, Nata, la Lupa, Peppa, si collocano alla periferia, pagando la loro 'diversità' con la vita o con l'emarginazione sociale e affettiva.

Grottesca ma emblematica summa delle maschere femminili verghiane è l'attricetta Aglae, sarcastico ribaltamento dell'immagine della femme fatale cara al Verga fiorentino che ne ribalta i connotati anche attraverso l'uso dissacrante di quei termini ("baiadera", "odalisca") che prima erano stati adoperati per connotarla ed esaltarla. Allorché il baronello Ninì Rubiera si reca dalla primadonna:

Un odore di stalla, in quella scaletta buia, cogli scalini unti e rotti da tutti gli scarponi ferrati del contado. Lassù in cima, un fil di luce, e una figura bianca, che gli si offrì intera, bruscamente, con le chiome sparse.

- Tu mi vuoi... baiadera... odalisca?..

C'erano dei piatti sudici sulla tavola [...].

All'uscio che metteva nell'altra stanza era inchiodato un bellissimo sciallo turco, macchiato d'olio; e dietro lo sciallo turco udivasi il signor Pallante che russava sulla sua gelosia.

Pochi, sapienti tratti sono sufficienti allo scrittore per delineare caratteri, situazioni, psicologie e stati d'animo carichi di un'energia e di una pregnanza che i fiumi $\mathrm{d}$ 'inchiostro generosamente sparsi negli affreschi giovanili non riescono ad eguagliare. Esotismo e mistero, lusso e fascinazione, mito e fantasia, si dissolvono e scompongono nel Verga maturo come i sogni di carta dei suoi vedi anni, spazzati via dalla fiumana inesorabile della vita e del progresso, conferendogli al contempo quell'incisività, icasticità e lirismo che costituiscono gli ingredienti segreti della sua grandezza.

\section{RIFERIMENTI BIBLIOGRAFICI}

AA.VV., Vergae il cinema, a cura di Nino Genovese e Sebastiano Gesù, Catania, Maimone, 1996 (in particolare i contributi di: Cardillo M., Donne verghiane tra pagina e film, ivi, p. 74; Fasanelli P., Tigre reale, “Cine-Fono", 11-25/11/1916, ivi, pp. 258-259, 1996; Zappia C., Una peccatrice, "Cronache dell' attualità cinematografica”, 30/1/1919, ivi, p. 264; Max, Eva, “La rivista cinematografica”, ivi, p. 266, 25/11/1920; Generosa G., La Lupa, “Rassegna del film”, n. 19, nov./dic. 1953, ivi, p. 294; Anonimo, L'amante di Gramigna, "Momento Sera", 8-9/2/1969, ivi, p. 305; Colombo F., La Lupa, “L'Eco di Bergamo", ivi, p. 323, 23/9/1996.

AA.VV., Giovanni Verga. Una biblioteca da ascoltare, Roma, Edizioni De Luca, pp. 41-88, 1999.

Brancaleoni F., "Le origini del tema della maschera: da Verga a Capuana. Linee di un percorso", in Critica Letteraria, n.3, pp. 551-582, 2001. 
Di Nallo A., “La zoologia dell'amore nel Verga fiorentino”, in Oliva G. (a cura di), Animali e metafore zoomorfe in Verga, Roma, Bulzoni, pp. 15-27, 1999.

Marchese D., La poetica del paesaggio nelle "Novelle rusticane" di Giovanni Verga, AcirealeRoma, Bonanno, pp. 300, 2009.

Martinelli V., Il cinema muto italiano. I film della grande guerra, 1916, Torino, Nuova Eri, 1992.

Oliva G. (a cura di), Verga G., Teatro, Milano, Garzanti, (vd.: Appendice sceneggiature cinematografiche), 1987.

Raya G., Verga e il cinema, Roma, Herder, 1984.

$\begin{array}{lllll}\text { Verga G., Mastro-don Gesualdo, Milano, Mondadori, } & 1979 .\end{array}$ ----, Tutte le novelle, a cura di C. Riccardi, Milano, Mondadori, 1984.

----, Una peccatrice, Storia di una capinera, Eva, Tigre reale, Milano, Mondadori, 1987.

Zaccaria G., Le maschere e $i$ volti. Il carnevale nella letteratura italiana dell'Otto-Novecento, Milano, Bompiani, 2003.

Zappulla Muscarà S., “Contributi per una storia dei rapporti tra letteratura e cinema muto (Verga, De Roberto, Capuana, Martoglio e la settima arte)", in La Rassegna della Letteratura Italiana, LXXXVI, (settembre-dicembre 1982); poi in Letteratura Teatro e Cinema, Catania, Tringale, pp. 217-310, 1984 e, ampliato, col titolo Giovanni Verga e il cinema muto, in AA.VV., Rilettura di Verga, a cura di R. Brambilla, Assisi, Pro Civitate Christiana, 1986.

----, “La Lupa" e l'annullarsi cosciente", in La Lupa. Novella, dramma, tragedia lirica, Palermo, Novecento, 1991.

Zappulla Muscarà S., "Giovanni Verga invisibile-burattinaio artista, fra teatro, melodramma e cinema", in Verga da vedere. Teatro. Cinema. Televisione, a cura di F. Caffo, S. Zappulla Muscarà, E. Zappulla, Palermo, Regione Siciliana, Assessorato ai Beni Culturali e Ambientali e della Pubblica Istruzione, pp. 244, 2003. 